Ceballos, E. (2014). Coeducación en la familia: Una cuestión pendiente para la mejora de la calidad de vida de las mujeres. Revista Electrónica Interuniversitaria de Formación del Profesorado, 17 (1), 1-14

DOI: http://dx.doi.org/10.6018/reifop.17.1.198811

\title{
Coeducación en la familia: Una cuestión pendiente para la mejora de la calidad de vida de las mujeres
}

\author{
Esperanza Ceballos \\ Universidad de La Laguna
}

\section{Resumen}

La vida cotidiana de las familias se plasma en usos del tiempo característicos de una socialización en función del género aún muy tradicional. No sólo las mujeres y las niñas siguen asumiendo mayor carga de trabajo familiar, encargándose más que los varones de las labores más pesadas y rutinarias, y de las tareas de cuidado de los miembros dependientes. Además, las madres disfrutan de menos tiempo de ocio y de peor calidad, en especial cuando ambos progenitores están empleados. Por fin, las madres trabajadoras tienden a sufrir consecuencias en su salud física y mental, experimentando más estrés e insatisfacción con sus parejas. Este aprendizaje sexista en la familia pone de relieve la insuficiencia de los actuales programas coeducativos, y plantea la necesidad de que la escuela se oriente no sólo a evitar el sexismo en las aulas, sino también a compensar el influjo de nuestra sociedad patriarcal.

\section{Palabras clave}

Género; trabajo familiar; ocio; coeducación.

\footnotetext{
Contacto

Esperanza Ceballos Vacas, eceballo@ull.edu.es, Área de Métodos de Investigación y Diagnóstico en Educación. Facultad de Educación (Módulo B). Universidad de La Laguna (Campus Central) Avda. Universidad s/n, 38204. La Laguna - Tenerife - Islas Canarias.

Este artículo se ha realizado en el marco del Proyecto EDU2012-38588: Implementación y evaluación del programa "Educar en Positivo" basado en entornos virtuales de aprendizaje para promover la parentalidad positiva. Ministerio de Economía y Competitividad.
} 


\title{
Coeducation in the family: An outstanding issue for improving the quality of life of women
}

\begin{abstract}
The daily life of families reveals a traditional use of time according to gender. Not only the women and the girls are still carrying on with a greater load of family-work, coping more than men with the heavier and more monotonous tasks, and with the caring of the dependent members. Furthermore, mothers are enjoying of less time for leisure; besides this leisure has got a worse quality because is often "contaminated", especially when both progenitors are employed. Finally, working mothers are likely to suffer consequences in their physical and mental health, experiencing more stress and dissatisfaction with their couples. This sexist socialization in the family points out to coeducation standards. School programs must be directed to objectives of coeducation much more ambitious, not only avoiding sexism, but compensating the negative influences still remaining in our patriarchal society.
\end{abstract}

\section{Key words}

Gender; family-work; leisure; coeducation.

\section{Introducción}

La conferencia Mundial sobre la Mujer celebrada en Beijing en 1995 supuso un hito en la historia de la humanidad al reclamar el fomento de políticas integradas que favorecieran el desarrollo de las mujeres. Esta declaración tan explícita sirvió para poner de manifiesto con toda rotundidad que, si bien se han producidos relativos avances en algunas zonas del planeta, continua existiendo una vergonzosa discriminación que afecta a la mitad de la población mundial (las mujeres). Y que para acabar con esta situación de injusticia es imprescindible que las directrices políticas queden impregnadas por una perspectiva de género dirigida a la reparación de estas desigualdades.

Sin duda, todo ello ha supuesto un nuevo impulso en las políticas de coeducación que se vienen preconizando en nuestro país, en especial a partir de la promulgación inicial de la LOGSE y de su mejora y evolución en la LOE. Los objetivos a desarrollar por las escuelas, entre otros, se han orientado a eliminar los estereotipos que reforzaran las desigualdades entre hombres y mujeres, suprimiendo todos aquellos sesgos androcéntricos, fundamentalmente plasmados en el curriculum oculto de las escuelas. Sin embargo, y tras más de dos décadas de implantación de estas políticas educativas, y a pesar de las energías desplegadas en torno a estas acciones, es penoso tener que concluir que, aunque se han obtenido logros no desdeñables, aún es preciso aunar más fuerzas para romper la barrera de las diferencias entre los hombres y las mujeres. En este sentido, Rodríguez (2007) apunta, entre otras, las siguientes razones que justifican la persistencia de las discriminaciones en el ámbito educativo:

- Escasa sensibilización en los socializadores sobre la existencia de desigualdad, por creer falsamente en el logro efectivo de la igualdad.

- Subsistencia de los usos androcéntricos en el lenguaje oral o escrito. 
- Permanencia de estereotipos de género en las prácticas pedagógicas.

- Invisibilidad de las mujeres en el conocimiento científico.

Parece pues imprescindible empezar por revisar la realidad cotidiana de los centros escolares y las familias. Porque por encima de las buenas intenciones que en general se predican (p.e. un discurso no sexista), es innegable que prevalecen las evidencias de lo que se hace (p.e. encomendar diferentes tareas a los niños en función de sus género) en las aulas y en las casas. Porque un proyecto para la igualdad entre hombres y mujeres no sólo implica su transmisión formal, sino que supone un reto mucho más ambicioso: trasladar las teorías coeducativas a las vivencias del día a día. Por todo ello, en este artículo me centraré en el análisis de una parte fundamental de la socialización: la familiar, incidiendo en el carácter diferencial de los usos del tiempo cotidiano en función del género, y en las consecuencias negativas que ello reporta en la calidad de vida de las mujeres.

\section{Educación en la familia y coeducación}

Que la familia y la escuela son las fuentes básicas de la socialización y que ambas deben contribuir al desarrollo integral del ser humano no parece requerir mayor explicación. La educación familiar desarrolla aprendizajes para la vida que nos deben enseñar cómo afrontar retos y asumir responsabilidades (Ceballos, 2006). Sin embargo, mientras que la educación escolar es objeto de sesudos debates que terminan por configurar un complejo entramado explicitado en forma de leyes, curriculum, proyectos... la educación familiar permanece más o menos oculta y autorregulada en la intimidad de los hogares. Así que, mientras que la educación escolar es un asunto de dominio público, la naturaleza de las prácticas educativas de cada familia pertenece al ámbito de lo privado. Por ello es imprescindible prestar atención a los datos que nos revelan lo que sucede en el seno de las familias. $Y$ son muchos los indicadores que nos muestran «el nivel coeducativo» de las familias a través del estudio de su vida diaria.

Para empezar, ya desde el propio nacimiento nuestra sociedad insiste en definir las características diferenciales de género de acuerdo con costumbres altamente imbricadas en la socialización (p.e. se reserva el color rosa y los pendientes para las niñas). Los adultos no sólo regulan de inmediato la apariencia de este nuevo ser humano, sino que con frecuencia también seleccionan el lenguaje (p.e. princesas y machotes), y los juegos para la interacción (p.e. con actividades en general más enérgicas para los varones) en función del sexo del bebé. Esta tradicional distinción por sexos que se emprende en la familia y en el grupo social de pertenencia está muy arraigada desde los primeros momentos de la vida del recién nacido, y continua su influjo a lo largo de la infancia marcando estereotipos de género. Por ejemplo, a los niños se les fomenta ser duros emocionalmente, heterosexuales y dominantes mediante la represión de las emociones, la exaltación de la homofobia y la ridiculización de lo femenino; y en las niñas ser coquetas, delicadas, sacrificadas y empáticas mediante la preocupación temprana por la imagen, un control más severo sobre la impulsividad verbal (p.e. las niñas no dicen tacos) y conductual (p.e. las señoritas no corren, no se meten en peleas... ), y la inmersión en la ética del cuidado. Rasgos que son reforzados a menudo por los medios de comunicación, y también muy frecuentemente por la escuela (sobre todo a través del curriculum oculto).

En suma, la construcción de la identidad de género se gesta desde la infancia de forma dinámica a través de la interacción diaria, asociando determinados rasgos y conductas a lo masculino y a lo femenino (Domínguez Folgueiras, 2009). Por eso los discursos grandilocuentes quedan pulverizados ante el poderoso aprendizaje vicario vehiculado a 
través de lo que los hijos e hijas ven hacer día a día a sus padres y a sus madres. Por ese motivo a continuación vamos a prestar atención a la presencia o ausencia de coeducación familiar en función de los modelos de socialización que padres y madres ofrecen con su ejemplo diario a sus hijos e hijas. De esta forma se harán patentes las notables discrepancias que actualmente subsisten en sus usos habituales del tiempo, moldeando una identidad diferencial en función del género: con más trabajo familiar y menos ocio para las madres que para los padres. Más allá de lo que esto significa como injusticia social, se enfatizarán las relevantes consecuencias de esta desigualdad en la calidad de vida de las mujeres, y por ende en la calidad de la convivencia familiar y en la propia estabilidad de los proyectos familiares.

\section{Socialización del trabajo familiar}

Estos estereotipos de género encuentran una de sus representaciones más genuinas en el reparto del llamado "trabajo familiar" (Maganto y Bartau, 2004) que muestra una socialización nítidamente sexista. Según datos recientes referidos por Meil (2006) "todavía no se ha llegado a una plena igualdad de trato y exigencia de colaboración a los hijos y a las hijas". De forma que entre los 10 y los 18 años algo más del 50\% de los hijos varones colaboran en las labores del hogar, frente al $76 \%$ de las hijas. Además el tiempo medio diario invertido por ellos (67 minutos) es inferior al invertido por ellas (93 minutos), con el agravante de que estas diferencias se hacen más patentes a medida que la edad avanza. Estas discrepancias dan un salto cualitativo cuando las mujeres inician la convivencia con su pareja y se reproducen los clásicos esquemas de división sesgada del trabajo familiar; hecho principalmente palpable en las parejas en las que ambos están empleados. Sin embargo, la exacerbación de las desigualdades entre los hombres y las mujeres, se provoca con la llegada de los hijos, acentuando el desequilibrio percibido por las mujeres (Milkie y Petrola, 1999).

Sin duda, la transición hacia la paternidad y la maternidad supone un cambio radical hacia una nueva etapa del ciclo vital en el que se incluyen una considerable acumulación de estrenadas ocupaciones y responsabilidades familiares. Estos retos de adaptación en las actividades del día a día deberían ser asumidos idealmente de forma paritaria entre ambos miembros de la pareja. No obstante, son notorios los innegables desajustes que persisten en el reparto equitativo de lo que se ha venido a llamar el trabajo familiar. En este punto parece pertinente dilucidar el concepto de trabajo familiar, aclarando sus diferencias con el más conocido como «trabajo doméstico». Si bien este último viene a formar parte del trabajo familiar, hay que puntualizar que el trabajo familiar alude a un concepto más amplio que engloba el esfuerzo y el tiempo dedicado al desarrollo y realización de las competencias necesarias para el mantenimiento del hogar, el cuidado de los hijos (u otros miembros dependientes) y su educación (Maganto, Etxeberria y Porcel, 2010).

Esta distinción es relevante pues los mismos autores establecen que el trabajo familiar incluye una diversidad de roles entre los que cabe distinguir al simple ejecutor de las tareas, del organizador que debe planificar, delegar y supervisar su correcto cumplimiento. Siendo este último rol tan complejo (pero invisible y por tanto no justipreciado) característico de la mujer. El hecho de que las estadísticas no tengan en cuenta este rol a la hora de contabilizar el número de horas invertidas es interpretado por Carrasco (2006) en función del interés de la sociedad patriarcal en mantener oculto el trabajo familiar realmente llevado a cabo por las mujeres. A ello se debe sumar que las mujeres suelen desempeñar ambos roles (organizador y ejecutor), dedicando tiempos y energías físicas y mentales ostensiblemente superiores a las de sus compañeros de vida. Que la división del trabajo familiar permanece 
descompensada incluso en los países más desarrollados parece fuera de discusión (Braun, Lewin-Epstein, Stier y Baumgärtner, 2008). Por ejemplo, Maganto, Bartau y Etxeberria (2003) concluyen que las madres soportan una importante sobrecarga de trabajo en relación con el resto de los miembros de la familia, manifestando el consecuente descontento con la contribución de los padres.

Ya en los años 90 el estudio pionero en España de Finkel (1997) señalaba que las madres empleaban más tiempo ( 5 horas y 48 minutos de media semanal) y energía que los padres (1 hora y 54 minutos) al trabajo familiar, apreciándose además una clara segregación de labores en función del género: por ejemplo, el $65.7 \%$ de las madres siempre hacían la compra, el $81.4 \%$ lavaban la ropa, el $63.4 \%$ llevaban a los hijos al médico, el $61.6 \%$ iban a hablar con los profesores de sus hijos... en contraste, un $44.4 \%$ de los hombres siempre se encargaban de hacer pequeños arreglos. Otros datos como los de Bianchi et al. (2000) revelan que las mujeres gastan casi el doble de tiempo que los hombres en el trabajo doméstico (17.5 horas semanales frente a las 10 de los hombres). Además las mujeres han sido educadas en una ética del cuidado que sitúa a las madres como principales agentes del cuidado de los hijos y las hijas. Tanto así que, según refiere Elósegui (2009), recogiendo datos del Observatorio para la igualdad de oportunidades entre hombres y mujeres, incluso en parejas con doble ingreso, las madres se hacen cargo de su atención en situaciones de enfermedad en un $72.5 \%$ de los casos, y en un $76.5 \%$ acuden a las reuniones escolares. A la luz de la Encuesta de Empleo del Tiempo 2009-2010 del INE, estos resultados no parecen haberse alterado demasiado dos décadas después. Las mujeres todavía ocupan cada día dos horas y cuarto más que los hombres en el trabajo familiar, sin tener en cuenta el tiempo, la dedicación y el consumo constante de energía mental que requieren las labores propias del rol de organizadora.

Este panorama de desigualdad puede generar en las mujeres una creciente insatisfacción que con frecuencia acabará derivando en un conflicto de mayor calado. La percepción de injusticia o no depende en gran medida del modelo de relación sostenido por las mujeres, de forma que las mujeres más tradicionales suelen sentirse más felices en sus relaciones ya que las expectativas sobre sus cónyuges son menos exigentes, y por tanto sus evaluaciones al respecto más positivas. Sin embargo, las mujeres con ideas más igualitarias se sienten más defraudadas, por lo que tienden a reclamar mayor apoyo de sus parejas encontrando a menudo una respuesta negativa (Wilcox y Nock, 2006).

Esta resolución inadecuada es la que definitivamente parece incrementar el conflicto inicial, pues como explican Johnson y Huston (1998), las mujeres han sido educadas para la renuncia a la individualidad y para la adaptación a las necesidades de sus compañeros. Una inversión de amor en beneficio de la salud emocional de su relación de pareja que generalmente es ignorada o incomprendida por los hombres. Sin embargo, el drástico incremento de tareas y responsabilidades que conlleva la llegada de los hijos puede sobrepasar la capacidad de las mujeres para hacer frente a tal sobrecarga, abocándolas a solicitar mayor corresponsabilidad familiar a los hombres. Es decir, que es el desbordamiento de funciones y quehaceres, y no tanto la desigualdad en sí, el que sirve de motor del conflicto; un conflicto que de obtener una resolución poco satisfactoria para las madres, promueve malestar y alejamiento afectivo de sus parejas (Grote, Clark y Moore, 2004).

En contrapartida, la percepción de mayor equidad en el reparto del trabajo doméstico mejora cuando los hombres son capaces de valorar y compartir las labores más ingratas y que tradicionalmente se atribuyen a las mujeres: ordenar, planchar, limpiar, lavar la ropa.... (Blair y Johnson, 1992). Estas tareas, clásicamente adjudicadas a las mujeres, suelen ser 
también las más frecuentes y reiterativas (por lo efímero de su resultado), lo que incrementa su invisibilidad (no se advierte su desempeño habitual, sino sólo su ausencia). También suele tratarse de las más infravaloradas (de «maruja») porque no se les presume dificultad ni necesidad de pericia (lo cual es ciertamente discutible), y además su prolijo cumplimiento es tildado de «manía» o absurdo afán de perfección, pues se consideran labores prescindibles (porque no se notan hasta que dejan de realizarse). Por consiguiente, no tienden a conllevar el mismo reconocimiento social que otros trabajos habitualmente imputados a los varones y que gozan de mayor visibilidad, y además son considerados como "más expertos" (de «manitas»): por ejemplo, el mantenimiento de un jardín, o la reparación de un electrodoméstico. Para que el varón emprenda con naturalidad y esmero las faenas «de maruja» es necesario que se posicione en contra del estereotipo masculino social imperante. Por ello, en el contexto de nuestra sociedad patriarcal, las mujeres también se encuentran más proclives a apreciar este esfuerzo adicional de sus compañeros.

Cuando tenemos en cuenta los datos más actuales que revelan que a partir de los 10 años de edad el $91.9 \%$ de las féminas realizan tareas domésticas y se ocupan del cuidado de las personas dependientes invirtiendo casi 4 horas y media semanales, frente al $74.7 \%$ de los varones que dedican unas 2 horas y media (INE, 2012), la conclusión parece indiscutible: a pesar de los esfuerzos coeducativos desarrollados por escuelas y campañas sociales, las evidencias indican la perseverancia de la desigualdad que opera en contra de las mujeres y las niñas en pleno siglo XXI. Por tanto, las madres de hoy y de mañana, que además trabajan dentro y fuera del hogar, soportan una carga vergonzosamente superior a la de los padres y los niños lo que, como a continuación veremos, actúa en detrimento de su desarrollo personal y social y de su salud física y mental.

\section{Socialización del ocio en la vida familiar}

Como contrapartida al trabajo retribuido y no retribuido (fundamentalmente el familiar) surge la necesidad de ocio como ámbito esencial del desarrollo humano que puede desplegarse en cualquiera de sus vertientes: lúdica, ambiental-ecológica, creativa, festiva, solidaria y económica (Liédana, Jiménez, Gargallo y Estévez, 2013). Lejos de entender el ocio como algo superfluo y vacuo, cada vez se entiende más como factor compensador del estrés derivado de las obligaciones diarias, y como elemento imprescindible para mejorar la salud, el bienestar y la felicidad. La necesidad de ocio adquiere más magnitud cuando se convierte en un bien escaso, lo que suele suceder en las familias de doble ingreso, complicando en especial sus posibilidades de ocio externo (Arnold y Lang, 2007). Lógicamente el tiempo dedicado al trabajo familiar resta posibilidades al de ocio, sobre todo alrededor de los 40 años que es cuando más se tienden a intensificar las labores de crianza. De hecho, Ekert-Jaffé (2010) calcula que el tiempo de dedicación a una familia con tres hijos equivale al de una ocupación laboral a tiempo completo, que puede o no ser compartido por la pareja.

En este punto hay que señalar que, aunque tanto los padres como las madres ven reducido su tiempo de ocio, una vez más la socialización de género impone un efecto diferencial en su disponibilidad. Pues de nuevo las madres se sienten impelidas a anteponer las necesidades de los miembros de su familia a las suyas propias. De hecho, Saxbe, Repetti y Graesch (2011) indican un orden de prioridad opuesto respecto al ocio y el trabajo doméstico en los padres ( $1^{\circ}$ ocio; $2^{\circ}$ actividades de comunicación; y $3^{\circ}$ trabajo doméstico) y en las madres ( $1^{\circ}$ trabajo doméstico; $2^{\circ}$ actividades de comunicación; y $3^{\circ}$ ocio). Por ello no es de extrañar que las mujeres sacrifiquen su exiguo tiempo libre adecuándose, por 
ejemplo, a los rígidos horarios de las actividades extraescolares de sus hijos, limitando así el uso de su tiempo personal y su flexibilidad (Lareau y Weininger, 2008).

Atendiendo a lo expuesto en el epígrafe anterior no sorprende que las mujeres, principalmente las que tienen hijos, sufran un déficit crónico de ocio, y que los hombres, en general, disfruten de casi hora y media más al día de ocio que ellas en EEUU (Mattingly y Bianchi, 2003). Esta desigualdad es también encontrada por Matud y del Pino (2011) en España comprobando que las mujeres invierten casi dos veces más tiempo en el cuidado de miembros dependientes y en las tareas caseras, y que los hombres gozan de más tiempo de ocio diario para salir con amistades, descansar y realizar otras actividades satisfactorias, subrayando que el peso de las diferencias de género es mayor en España que en otros países desarrollados. Este último resultado es corroborado por los últimos datos aportados por el INE (2012): los hombres españoles se permiten más actividades de ocio y durante más tiempo que las mujeres. Así, por ejemplo el $42.7 \%$ de los hombres invierten unas 2 horas a los deportes y a las actividades al aire libre, y el $35.6 \%$ otras dos horas con las aficiones y la informática, frente al $36.9 \%$ y $23.9 \%$ respectivamente de las mujeres que sólo conceden algo más de hora y media a estos mismos entretenimientos.

Pero no solo se trata de que las mujeres dispongan de menos tiempo personal, sino de que también poseen menos espacios privados, y merecen menos consideración respecto a las actividades que a ellas les gustan y les hacen sentir bien (Stalp y Conti, 2011), y que son juzgadas como más frívolas e innecesarias (p.e. comunicarse con sus amigas). En otras palabras: el ocio de los hombres se pone menos en discusión y es más respetado, mientras que el ocio de las mujeres es más trivializado y más fácilmente sacrificable en favor del «bienestar familiar». Con frecuencia las mujeres se hallan en el dilema de elegir entre su bienestar o el de sus hijos y su pareja, y cuando vencen a la presión social y optan por cuidarse a sí mismas a menudo experimentan sentimientos de culpa (Sánchez-Herrero, 2008). Todo ello se explica, sin duda, por el hecho de que las mujeres son imbuidas desde su infancia en esa ética del cuidado a la que he aludido anteriormente.

La solución de muchas mujeres trabajadoras a este callejón sin salida es intentar ejercer un poco más de «supermujeres» solapando las tareas de ocio a las del trabajo familiar; por ejemplo, viendo la televisión mientras planchan la ropa, o elaborando de cabeza la lista de la compra mientras hacen deporte. Este ejercicio mental es uno más dentro de los propios del rol de organizadora; un rol que ya de por sí es imperceptible en las estadísticas, y que además puede camuflarse contabilizado como tiempo efectivo de ocio de las mujeres. Aunque este binomio ocio-trabajo aligere la conciencia de las mujeres también produce las correspondientes consecuencias en la calidad del ocio. Por eso esta última autora insiste en que las mujeres no sólo tienen menos tiempo libre que los varones, sino que además ese insuficiente tiempo se encuentra más contaminado por otras actividades no ociosas. Además, de que se disfruta en intervalos más fragmentados (p.e. la lectura de una novela interrumpida constantemente por las demandas de los niños). Por otra parte, resalta que las madres suelen compartir más sus instantes de ocio con los hijos, mientras que los padres tienden más a excluir específicamente la presencia de los niños para consagrarse a un ocio «puro» con otros adultos.

En suma, aunque la vida de las mujeres actuales haya cambiado radicalmente con su incorporación masiva al mercado laboral, la socialización sigue marcando barreras invisibles para el ocio de las mujeres. Las madres se sienten obligadas a la dureza de una doble jornada real de trabajo pagado y no pagado, haciendo gala de auténticas destrezas malabares para poder atender adecuadamente su carrera laboral y la desproporción de sus obligaciones familiares. Arañar de esta doble jornada minutos para el crecimiento personal 
en forma de ocio supone ya una «pirueta triple» que pone en jaque la famosa competencia policrónica de las mujeres. Y es más, las mujeres pueden ser capaces hasta cierto punto de llevar a cabo multitareas, pero no debe obviarse el coste para su salud física y mental.

\section{Vida familiar y calidad de vida de las mujeres}

Como se ha ido desgranando en lo anterior, la vida familiar de las madres empleadas es sensiblemente diferente a la de los padres empleados. Las jornadas de las madres trabajadoras configuran un complicado puzle de actividades y responsabilidades que requieren una maratoniana y compleja gestión del tiempo. Aunque el papel de otros estresores crónicos (p.e. el tráfico vial) haya sido objeto de estudio, la influencia de la experiencia subjetiva del tiempo sobre el bienestar ha sido relativamente descuidada (Roxburgh, 2004). Esta ausencia de interés podría ser interpretada desde una perspectiva de género ya que, como apunta esta autora, la presión del tiempo se relaciona mucho más con la depresión en las mujeres asalariadas que en los hombres asalariados.

De entrada podemos sugerir la probable conexión de la depresión femenina en las madres trabajadoras con el estrés generado por su doble jornada, agravado por la escasez y baja calidad de su tiempo de ocio. Aparentemente mientras los padres usan su tiempo de ocio para relajarse y reponerse del estrés diario, las madres siguen ajetreadas y enfocadas en la organización del hogar y el cuidado de los hijos. Estas diferencias traducidas a términos biológicos implican que mientras los padres consiguen rebajar los niveles de cortisol (hormona del estrés) mediante períodos suficientes de ocio puro, las mujeres continúan manteniendo elevados niveles de esta hormona (Saxbe et al, 2011). Estos mismos autores explican que el cortisol muestra un ritmo diurno, elevándose al despertar por las mañanas para ir disminuyendo a lo largo del día. Esta caída máxima al ir concluyendo la jornada se relaciona positivamente con la recuperación fisiológica del estrés diario, lo que suele suceder en el caso de los varones. Sin embargo, en el de las mujeres la ausencia de ese declive aumenta las posibilidades del síndrome de burn-out, la depresión, e incluso la mortalidad prematura, según refieren estos últimos autores citando a Sephton, Sapolsky, Kraemer y Spiegel (2000).

Pueden ser muchos los factores que incidan en esta activación constante del estrés en las madres trabajadoras. El origen de esta desazón cotidiana no sólo se halla en las discrepancias evidentes que hemos encontrado en los usos del tiempo. Pues como oportunamente apunta Durán (2007: 219):

"las encuestas de uso del tiempo son herramientas muy circunspectas y no entran en los estados emocionales, así que no preguntan sobre los sentimientos de los entrevistados mientras dedican su tiempo a unas actividades y a otras. Por eso no sabemos con exactitud, sino por aproximación, el tiempo que dedicamos cada día a hacer unas risas, aburrirnos o llorar".

Es decir, que no sólo debemos considerar el tiempo que las madres dedican al trabajo familiar y al ocio, sino que también parece primordial dar cuenta de los estados emocionales que acompañan a la realización de estas actividades. En este sentido, hay que rescatar los estudios que indican el efecto de mayor depresión en mujeres que en hombres cuando invierten un tiempo similar al trabajo doméstico (Roxburgh, 2004). Y es que, como ya se ha destacado, las faenas adjudicadas a las mujeres (p.e. pasar la aspiradora o fregar el suelo) suelen ser más rutinarias y absorbentes. Pero, sobre todo, carecen de un punto claro de inicio y de finalización por la fugacidad de su resultado, promoviendo la frustración propia de una labor jamás inacabada, que además tiende a llevarse a cabo con emociones 
negativas (ansiedad por las prisas, rechazo por su inaceptación, y rabia por la percepción de injusticia). Sin embargo, las clásicas ocupaciones masculinas (p.e. el bricolaje casero) suelen obtener mayor recompensa en forma de tarea cumplida y finiquitada, elogios y reconocimiento social.

Para paliar esta sobrecarga física y emocional de las madres trabajadoras las familias con más posibilidades económicas suelen recurrir al empleo doméstico. Prácticamente siempre es otra mujer (la empleada doméstica) la que concede un alivio a las madres estresadas, permitiéndoles librarse al menos de una parte del trabajo familiar más pesado y repetitivo. A través de este subterfugio que esquiva la verdadera corresponsabilidad familiar, a menudo las mujeres experimentan una sensación falsa de mayor equidad. Pues como señalan Braun, Lewin-Epstein, Stier y Baumgärtner (2008) la disminución del trabajo familiar aumenta la percepción de justicia en su reparto. Sin embargo, esta aparente solución encierra una nueva vulneración de los derechos de las mujeres (Pérez Orozco, 2010) y un refuerzo de la socialización sexista, manteniendo intacta la segregación sexual de las tareas familiares.

Podría decirse que las mujeres también se «autoengañan» con esta suerte de arreglo. Porque, aparte de que el apoyo remunerado femenino constituye sólo una liberación parcial para las madres, también redunda en la creación de nuevas obligaciones. Las consecuentes demandas de coordinación para la logística doméstica de nuevo son atribuidas a las madres que una vez más deben delegar, supervisar y planificar las tareas compartidas con su nueva compañera de fatigas. Por tanto, mientras que la cuantía y la calidad de la contribución de los varones permanece intacta, el aumento del bienestar de las madres trabajadoras es solo relativo. Todo ello implica una tremenda hipocresía del sistema patriarcal que en realidad sale indemne de la reclamación femenina de una igualdad efectiva entre los hombres y las mujeres.

Por otra parte, es probable que las madres con ideas igualitarias vean aún más empañada su calidad de vida pues ellas son conscientes de su desigualdad, y de la incoherencia manifiesta entre sus creencias paritarias y su realidad cotidiana. De hecho, estas madres experimentan más culpa y resentimiento, y mayor insatisfacción con sus parejas con respecto al reparto del trabajo familiar (Ogletree, Worthen, Turner y Vickers, 2006). Así su doble o triple jornada se suma a su insatisfacción personal, que se combina peligrosamente con la decepción al evaluar a sus parejas. Todos los ingredientes para la receta del burn-out y la depresión aderezada por un creciente malestar en su relación de pareja (Voydanoff y Donnelly, 1999). Ya que son ellas quienes se hallan en el lado más pernicioso de la balanza, es natural que se muestren más sensibles con los conflictos relacionados con el trabajo familiar y promuevan discusiones para intentar corregir esa injusticia. Sin olvidar que la calidad emocional de un matrimonio es un predictor de divorcio más potente en las mujeres que en los hombres (Sayer y Bianchi, 2000), y que justamente el principal predictor de divorcio en los primeros siete años de convivencia es la falta de respuesta positiva y afectiva de los varones ante los conflictos del día a día (Gottman y Levenson, 2000).

Las consecuencias en la calidad de vida de las mujeres presentes y futuras no son una cuestión baladí. Las dificultades de las madres para conciliar su vida familiar, laboral y personal las exponen a un mayor riesgo psicosocial que es pernicioso para su salud: el $53.4 \%$ de las mujeres frente al $27.2 \%$ de los hombres (Catalina, Corrales, Cortés y Gelpi, 2008). En palabras de Mazarrasa y López-Doriga (2006: 2) las mujeres afrontan «una división emocional entre las exigencias del empleo y las necesidades de la familia, generando con frecuencia una doble culpabilidad, aumentando la predisposición a enfermar o a cronificar [sic] 
dolencias y a sufrir accidentes». En otras palabras, la salud de las madres trabajadoras se encuentra seriamente comprometida por la desigualdad de género en su vida cotidiana.

\section{Conclusiones}

El ideario coeducativo ha logrado implantarse más en el plano de lo formal que en el de lo real, creando una ilusión de falsa igualdad ampliamente extendida (Rodríguez, 2007). Por ello, y a pesar de los gestos triunfalistas de igualdad, la socialización discriminatoria en función del género permanece vigente tras décadas de coeducación. Las evidencias no dejan lugar a las dudas: cuando atendemos a la realidad privada de las familias es innegable que la sociedad patriarcal continúa reproduciendo su esquema clásico de división sexual del trabajo en la intimidad de los hogares. Un aprendizaje vicario que se transmite cotidianamente a los hijos y a las hijas en los modelos diferenciales de sus padres y sus madres.

Las madres, sobre todo en parejas de doble ingreso, se encuentran atrapadas por su doble jornada (trabajando dentro y fuera de casa), y siguen invirtiendo su tiempo y sus energías en atender las necesidades materiales (económicas) y organizativas de su familia (planificar, gestionar, delegar, supervisar y ejecutar el trabajo familiar). El tiempo que les resta para disfrutar de un ocio reparador es escaso y de baja calidad pues: se encuentra a menudo contaminado por otras acciones no ociosas que discurren en paralelo; está fragmentado en tiempos más cortos porque queda relegado por la priorización de las obligaciones familiares o por el ocio del resto de la familia; y, además, es menos renovador porque esa auto-concesión suele implicar una dosis de culpabilidad materna.

Es cierto que los padres varones van avanzando tímidamente hacia el camino de la igualdad, pero aún queda un largo trecho por recorrer. Con frecuencia las estadísticas ofrecen cifras que reducen distancias en las discrepancias de los tiempos del trabajo familiar y del ocio de los padres y las madres. Sin embargo, las medidas de uso del tiempo no parecen instrumentos lo suficientemente sensibles (Parra, Ceballos, Fontana y Bartau, 2012) para captar las verdaderas diferencias. Pues las horas y los minutos empleados no informan del tiempo y la energía mental consumidos por las organizadoras de la logística familiar. Tampoco discriminan si la disminución en el tiempo gastado en las labores familiares puede ser el resultado de la velocidad y el estrés, y/o del solapamiento de tareas para ajustar la doble jornada de las madres. Por último, tampoco describen las emociones con las que se viven las actividades de trabajo y de ocio. No reparan en si las madres se sienten frustradas, nerviosas y enfadadas mientras ordenan y limpian la casa, o en si se sienten culpables y egoístas mientras descansan o se divierten.

Lo que sí sabemos es que la calidad de vida de las madres presenta un riesgo psicosocial superior al de los padres (Catalina et al, 2008), especialmente en las familias de doble ingreso. El estrés constante y la falta de desconexión con las responsabilidades familiares abocan a las madres trabajadoras a sufrir consecuencias negativas en su salud (Mazarrasa y López-Doriga, 2006), que se relacionan con la permanente activación de los niveles de cortisol, que a la postre favorecen la aparición del estrés, la depresión y la mortalidad prematura (Sephton et al, 2000). Por lo tanto, no sólo se encara una tremenda injusticia social, con frecuencia también ridiculizada como «las pesadas lamentaciones esclavistas de las madres». La importancia de esa constante reclamación de igualdad y visibilidad adquiere tintes de mayor gravedad porque su efecto se extiende hacia la salud física y mental de las mujeres. 
Mientras tanto se prorroga la socialización coeducativa o no coeducativa de forma autorregulada en cada familia. Las mujeres con ideas más igualitarias perciben una inaceptable desigualdad y se sienten insatisfechas con su vida familiar. Pero las posibilidades de negociación familiar están limitadas porque las familias carecen de las herramientas necesarias para alcanzar un consenso. Las familias no pueden negociar porque no saben dialogar y argumentar poniéndose en el lugar de los demás (Correa et al, 2009). Además es muy posible que los padres y las madres perciban realidades diametralmente opuestas que les impidan partir de algún elemento compartido para iniciar el debate: por ejemplo, una mujer puede quejarse de soportar una mayor carga de trabajo, y su pareja puede mostrarse perpleja sin comprender el motivo del conflicto, convencido de que asume su mitad.

En este punto se enfatiza la importancia de reforzar los programas coeducativos de forma que lleguen al seno de las familias. Sin embargo hemos de reconocer que, ante la persistente dificultad para acceder a este ámbito tan privado, hemos de seguir confiando en la escuela, aunque siga siendo sexista en su cotidianeidad (Rodríguez, 2007). En este sentido, Díaz-Aguado y Martín Seoane (2011) insisten en que la escuela debe realizar esfuerzos mucho mayores, no solo descartando una socialización sexista, sino también contrarrestando el peso social del arraigo de una división ancestral. El sistema educativo, si bien insuficiente para desterrar las desigualdades de género, es una pieza esencial para el cambio (Subirats, 1999).

\section{Bibliografía}

Arnold, J. y Lang, U. (2007). Changing American home life: trends in domestic leisure and storage among middle-class families. Journal of Family Economical Issues 28, 23-48.

Bianchi, S.M., Milkie, M.A., Sayer, L.C. y Robinson, J.P. (2000). Is anyone doing the housework? Trends in the gender division of household labor. Social Forces 79(1), 191-228.

Blair, S. y Johnson, M. (1992). Wives' perceptions of the fairness of the division of household labor: the intersection of housework and ideology. Journal of Marriage and the family, 54, 570-581.

Braun, M., Lewin-Epstein, N., Stier, H. y Baumgärtner, M.K. (2008). Perceived equity in the gendered division of household labor. Journal of Marriage and Family 70, 1145-1156.

Carrasco, C. (2006). La paradoja del cuidado: Necesario pero invisible. Revista de Economía Crítica 5, 39-64.

Catalina, C., Corrales, H., Cortés, M.V. y Gelpi, J.A. (2008). Las diferencias de género en el nivel de exposición a riesgos psicosociales entre trabajadores/as de la comunidad Autónoma de Madrid: resultados preliminares del plan de investigación del estrés laboral de Ibermutuamur. EduPsykhé: Revista de psicología y psicopedagogía 7(2), 185-200.

Ceballos, E. (2006). Dimensiones del análisis del Diagnóstico en Educación: El diagnóstico del contexto familiar. Relieve, 12(1), 33-47.

Correa, N.; Rodríguez, J.; Batista, L.; Padrón, I. y Ceballos, E. (2009). Discurso argumentativo en episodios de conflictos entre padres e hijos adolescentes. Infancia y Aprendizaje 32, $467-484$. 
Díaz-Aguado, M.J. y Martín Seoane, G. (2011). Convivencia y aprendizaje escolar en la adolescencia desde una perspectiva de género. Psicothema 23(2), 252-259.

Domínguez Folgueiras, M. (2009). La división del trabajo doméstico en las parejas españolas. Un análisis del uso del tiempo. Revista Internacional de Sociología, 70(1), 153-179.

Durán, M. Á. (2007). El valor del tiempo ¿Cuántas horas te faltan al día? Madrid: Espasa.

Ekert-Jaffé, O. (2010). Are the real time costs of children equally shared by mothers and fathers? Social Indicators Research 101, 243-247.

Elósegui, M. (2009). Educar en la corresponsabilidad entre mujeres y hombres. La igualdad necesaria. Revista General de Derecho Canónico y Derecho Eclesiástico del Estado, 20, $1-29$.

Gottman, J. y Levenson, R. (2000).The timing of divorce: predicting when a coupe will divorce over a 14-year period. Journal of Marriage and the family 62, 737-745.

Grote, N. K., Clark, M. S. y Moore, A. (2004). Perceptions of Injustice in Family Work: The Role of Psychological Distress. Journal of Family Psychology 18(3), 480-492.

INE (2012). Encuesta de empleo del tiempo 2009-2010.

Johnson, E.M. y Huston, T.L. (1998). The perils of love, or why wives adapt to husbands during the transition to parenthood. Journal of marriage and the family, 60, 195-204.

Lareau, A. y Weininger, E.B. (2008). Time, work, and family life: reconceptualizing gendered time patterns through the case of children's organized activities. Sociological Forum, 23(3), 419-454.

Liédana, L., Jiménez, T.I., Gargallo, E. y Estévez, E. (2013). Guía de ocio en familia. El tiempo que pasamos juntos. Madrid: Pirámide.

Maganto, J. y Bartau, I. (2004.) Corresponsabilidad familiar. Fomentar la cooperación y responsabilidad de los hijos. Programa COFAMI. Madrid: Pirámide.

Maganto, J., Bartau, I. y Etxeberría, J. (2003). La participación en el trabajo familiar: Un reto educativo y social. Relieve, 9, 2, 160-183.

Maganto, J., Etxeberría, J. y Porcel, A, (2010). La corresponsabilidad entre los miembros de la familia como factor de conciliación. Educatio Siglo XXI, 28, 69-84.

Mattingly, M. y Bianchi, S.M. (2003). Gender differences in the quantity and quality of free time: the U.S. experience. Social Forces 81(3), 999-1031.

Matud, P. y del Pino, M.J. (2011). Relevancia de la profesión en los usos del tiempo de mujeres y hombres. Salud de los trabajadores 19, 135-146.

Mazarrasa, L. y López-Doriga, B. (2006): La salud laboral desde la perspectiva de género". Seminario sobre "Prevención de riesgos de las mujeres en el trabajo". UIMP: Santander.

Meil, G. (2006). Padres e hijos en la España actual. Colección Estudios Sociales. Barcelona: Obra Social la Caixa.

Milkie, M. A. y Peltola, P. (1999). Playing all the roles: gender and work-family balancing act. Journal of Marriage and the family 61, 476-490. 
Parra, J., Ceballos, E., Fontana, M. y Bartau, I. (2012). Familia y educación en una sociedad en red. En C. Jiménez, García, J.L., Álvarez, B. y Quintanal, J. (coords). Investigación y Educación en un mundo en red. McGraw-Hill: Madrid.

Pérez Orozco, A. (2010). Insostenibilidad del sistema global de cuidados y alternativas feministas. Mujeres, sexo, poder, economía y ciudadanía. XX Taller de Política Feminista. Forum de Política Feminista 20, 11-22.

Rodríguez, V. (2007). La educación como ámbito prioritario de aplicación de la transversalidad de género. En A. Vega (coord.) Mujer y educación. Una perspectiva de género (pp. 15-30). Málaga: Aljibe.

Roxburgh, S. (2004). There just aren't enough hours in the day: the mental health consequences of time pressure. Journal of Health and Social Behavior 45, 115-131.

Sánchez Herrero-Arbide, S. (2008). La importancia de la perspectiva de género en la psicología del ocio. Anales de Psicología 24(1), 64.

Saxbe, D.E., Repetti, R.L. y Graesch, A.P. (2011). Time spent in housework and leisure: links with parents' physiological recovery from work. Journal of Family Psychology 25(2), 271-281.

Sayer, L. C.y Bianchi, S. M. (2000). Women's Economic Independence and the Probability of Divorce. Journal of Family Issues 21(7), 906-943.

Septon, S., Sapolsky, R., Kraemer, H. y Spiegel, D. (2000). Diurnal cortisol rythm as a predictor of breast cancer survival. Journal of National Cancer Institute, 92, 994-100.

Stalp, M. y Conti, R. (2009). Serious leisure in the home: professional quilters negotiate family space. Gender, work and organization, 18(4), 399-414.

Subirats, M. (1999). Género y escuela. En C. Lomas (comp.) ¿lguales o diferentes? Género, diferencia sexual, lenguaje y educación. Barcelona: Paidós.

Voydanoff, P. y Donnelly, B. (1999). The intersection of time in activities and perceived unfairness in relation to psychological distress and marital quality. Journal of Marriage and the Family 61, 739-751.

Wilcox, W. y Nock, S. (2006). What's love got to do with it? Equality, equity, commitment and women's marital quality. Social Forces 84(3), 1321-1345. 


\section{Autores}

Esperanza Ceballos Vacas

Profesora Titular de Universidad del Área de Métodos de Investigación y Diagnóstico en Educación. Mis líneas de investigación principales son: la investigación, evaluación y diagnóstico educativos, la convivencia familiar y escolar, y el riesgo social. Actualmente soy miembro activo del Proyecto EDU2012-38588: Implementación y evaluación del programa "Educar en Positivo" basado en entornos virtuales de aprendizaje para promover la parentalidad positiva 